Biophysical Chemistry 9 (1979) 345-353

c) North-Holland Publishing Company

\title{
A ROTATIONAL DIFFUSION COEFFICIENT OF THE 7OS RIBOSOME DETERMINED BY DEPOLARIZED LASER LIGHT SCATTERING
}

\author{
I. BRUINING and H.MV. FIJNAUT \\ Department of Molecular Cell Biology, Molecular Biology Section and \\ Van 't Hoff Laboratory for Physical and Colloid Chemistry, \\ University of Utrecht, Utrecht, The Netherlands
}

Received 22 August 1978

Revised manuscript received 15 December 1978

\begin{abstract}
We have obtained a rotational diffusion coefficient of the 70S ribosome isolated from Escherichio-coli (MRE-600), from the depolarized light scattering spectrum measured by photon correlation spectroscopy. The intensity correlation function of depolarized scattered light contains contributions due to multiple scattered and anisotropy scattered light from the ribosomal particle. We discuss extensively the subtraction procedure used to obtain the rotational correlation time from the experimental correlation function. We have also obtained the translationai diffusion coefficient from the same sample by deterriining the polarized correlation function. The hydrodynamic radius determined from the rotational diffusion coefficient is only slightly larger than the radius obtained from the translational diffusion coefficient. Therefore the ribosomal particle has a non-spherical shape. This conclusion. however, could be impaired by the effect of free drainin? of the ribosome.
\end{abstract}

\section{Introduction}

Hydrodynamic methods are widely used to characterize the shape and size of macromolecules. These methods have recently received much interest, as it has become possible to measure the translational diffusion coefficient by laser light scattering as a matter of routine procedure using photon correlation spectroscopy (PCS) [1]. When the translational diffusion coefficient of ribosomal particles was determined by laser light scattering, it was found that the hydrodynamic volume $\left(\frac{4}{3} \pi a_{0}^{3}\right)$ obtained by applying the Stokes-Einstein equation $\left(D=k_{\mathrm{B}} T /\right.$ $\left.6 \pi 3 a_{D}\right)$ was very much larger than the dry volume [2]. Therefore it has been concluded that the ribosomal particle contains large amounts of specifically hydrated and more or less trapped water. From a measurement of the rotational diffusion coefficient in addition to the translational diffusion coefficient one can determine the deviation from the spherical shape [3] and the hydrated size of the ribosomal particle.

Depolarized light scattering methods have been applied to determine the rotational diffusion coeffi- cient of small proteins, using Fabry-Perot interferometry [3], and of very large molecules with dimensions s of order $\lambda_{m}$ (wavelength of light in medium), such as tobacco mosaic virus, using PCS $[4,5]$. Therefore it is interesting to test also the applicability of PCS to determine the rotational diffusion coefficient of particles small compared to $\lambda_{\mathrm{m}}$, e.g. ribosomal particles.

The intensity of depolanized scattered light from macromolecules is usually three to four orders of magnitude smaller than the intensity of polarized scattered light [6]. This means that in practice the depolarized scattered signal suffers from a low predetection signal to noise ratio [6]. This ratio can be improved $(i)$ by measuring at very small $\left(\theta \approx 2^{\circ}\right)$ or very large $\left(\theta \approx 178^{\circ}\right)$ scattering angles, (ii) by slowing down the rotational motion by increasing the viscosity of the solvent, and (iii) by observing the scattered light from concentrated solutions of macromolecules.

From the depolarized light scattering spectrum, one can calculate the rotational relaxation time [7], which is related to the rotational diffusion coefficient. By applying the Debye model [8] one can calculate 
the volume of a sphere having the same rotational diffusion coefficient as the macromolecule. By comparing this hydrodynamic volume to the volume of a sphere which has the same transiational diffusional coefficient as the macromolecule one can establish whether the deviation from the spherical shape is large [9]. Complications may occur if hydrodynamic and dry volume are different and the particle is party flushed by the solvent $[10,11]$.

\section{Experimental}

A hundred grams of Escherichia-coli MRE-600, a generous gift of the University of Leiden, were harvested in midlog phase washed in standard buffer

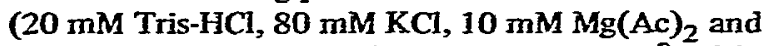
$6 \mathrm{mM}$ 2-mercaptoethanol) and stored at $-80^{\circ} \mathrm{C}$. The ribosomal pellet $105000 \mathrm{~g}$ which was obtained by standard procedures [12] was resuspended in standard buffer I ( $50 \mathrm{mM}$ Tris- $\mathrm{HCl}, 50 \mathrm{mM} \mathrm{KCl}, 10 \mathrm{mM}$ $\mathrm{Mg}(\mathrm{Ac})_{2}$ and $6 \mathrm{mM}$ 2-mercaptoethanol, $\mathrm{pH}$ 7.6). To this buffer $\mathrm{NH}_{4} \mathrm{Cl}$ was added to a final concentration of $1 \mathrm{M}$. The ribosomes were washed by layering the suspension on top of a $0.9 \mathrm{M}$ sucrose solution containing $1 \mathrm{M} \mathrm{NH}_{4} \mathrm{Cl}$ in addition to buffer $\mathrm{I}$ and centrifuging for 3 hours at $60.000 \mathrm{rpm}$.

The $70 \mathrm{~S}$ ribosomes were further purified by zonal centrifugation and collected by sedimentation. The ribosomal pellet was resuspended in buffer I which was made dust-free by filtration through millipore filters. The $70 \mathrm{~S}$ ribosomes were analyzed on glycerol gradients before and after the experiments to determine whether denaturation had occurred in vitro.

One day before use the ribosomes were dialyzed overnight against a glycerol solution in buffer $\mathbf{I}$. By comparing the refractive index of the dialyzate and ribosomal solution, we checked whether the dialysis had been complete. The concentrations used were in the range 0.012 to $0.020 \mathrm{~g} \mathrm{~cm}^{-3}$ as has been measured by UV spectroscopy [13] at $\lambda=260 \mathrm{~nm}$. The scattering cell with ribosomal solution was centrifuged for three hours at $4000 \mathrm{rpm}$ in order to pellet the dust particles. In spite of these precautions, dust was occasionally a disturbing factor for observing depolarized scattering at very small scattering angles.

For the light scattering experiments we isit the light from an argon ion laser (Spectra Physics model

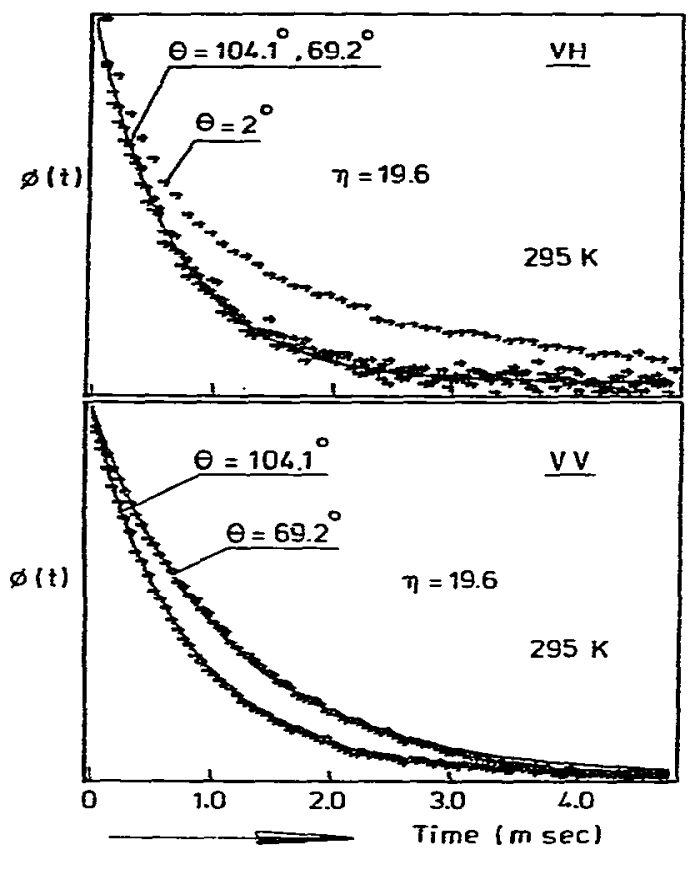

Fig. 1 . Comparison between tize polarized (VV) and depolarized $(\mathrm{VH})$ correlation functions $\phi(t)$ observed at various scattering angles. Note that the VH scattering is much less dependent on the scattering angle. The viscosities are expressed in $\mathrm{g} \mathrm{m}^{-1} \mathrm{~s}^{-1}$.

165) polarized by a Glann-Thompson prism (polarizer) perpendicular to the scattering plane.

The laser operated at $514.5 \mathrm{~nm}$ with an output power of $1 \mathrm{~W}$ or less. The polarized incident light was focussed into the scattering cell of $3 \times 1 \times 1 \mathrm{~cm}^{3}$ made of optical special glass (HELLMA). The windows of the cell did not scramble the polarized light to a noticeable degree. A Glann-Thompson prism (analyzer) was inserted in the scattered beam to distinguish either the radiation with polarization vector perpendicular to (VV) or parallel (VH) with the scattering plane. The extinction ratio of our polarizing prisms was determined to be of the order $10^{-6}$ by measureing the transmission of the laser light in forward jirection in the VH and VV configuration. The alignment was slightly dependent on the setting of the analyser as the front and back of the G-T prism were not exactly parallel.

The output of the detector was analyzed by a Saicor 
42A correlator operating in photon counting mode. In this way we obtained the correlation function $\langle n(t) n(0)\rangle$ where $n(t)$ is the number of photons counted in a small sample interval around $t[14]$, and the brackets denote an ensemble average.

In fig. 1 we have shown correlation functions $\phi(t)$ defined by

$\phi(t)=\left\{\langle n(t) n(0)\rangle-\langle n)^{2}\right\} /(n)^{2}$

for polanzed (VV) and depolarized (VH) scattering at two scattering angles. Note that the depolarized correlation function is much less dependent on scattering angle than the polarized correlation function.

At small scattering angles one observes that the depolarized correlation functions are not single exponentials and depend on the viscosity of the solvent: see fig. 2 .

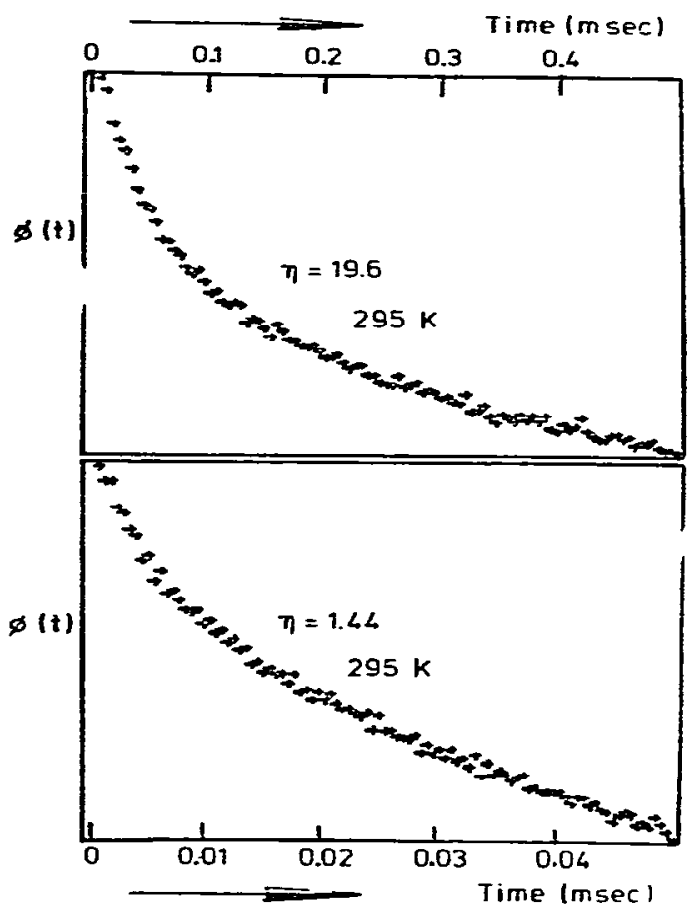

Fig. 2. Experimental VH correlation functions $\phi(t)$ obtained at two viscosities. The correlation functions show a similar behavior at different time scales. Viscosities in $\mathrm{g} \mathrm{m}^{-1} \mathrm{~s}^{-1}$.

\section{Theory}

Our aim is to characterize ribosomal particles by measuring the normalized field autocorrelation functions $c_{\mathrm{VH}}(t)$ and $c_{\mathrm{VV}}(t)$ for $\mathrm{VH}$ and $\mathrm{VV}$ configurations respectively.

For dilute particle concentrations, $c_{\mathrm{Vv}}(t)$ is given by [15]

$c_{* r v}(t)=\exp \left(-D k^{2} t\right)$,

where $D$ is the translational diffusion coefficient, $k=$ $\left(4 \pi / \lambda_{m}\right) \sin (\theta / 2)$, and $\theta$ the angle between incident and scattered light. The interpretation of $D$ can only be specified if a model for the particle geometry is chosen. In the case of spherical particles, $D$ is given by the equation: $D=k_{\mathrm{B}} T /\left(6 \pi \eta a_{\mathrm{D}}\right)$, where $\eta$ is the solution viscosity and $a_{\mathrm{D}}$ the hydrodynamic radius of the particles for translational diffusion.

For dilute particle concentrations $c_{\mathrm{VH}}(t)$ contains information about the relaxation of optical anisotropies [16]. We shall only consider the case that these optical anisotropy relaxations are a consequence of the reorientational motion of the molecule [17]. If we assume that translational and rotational motions are uncoupled, $c_{\mathrm{VH}}(t)$ can be expressed as:

$c_{\mathrm{VH}}(t)=c_{\mathrm{R}}(t) \exp \left(-D k^{2} t\right)$

where $c_{\mathrm{R}}(t)$ denotes the anisotropy scattering contribution. The complexity of the expressions [18] for $c_{\mathrm{R}}(t)$ arises from the possibility that the principal axes for the optical anisotropy does not coincide with the principal axes of the rotational diffusion and the fact that for arbitrarily shaped particles the rotational diffusion coefficients and the polarizabilities along the principal axes do not have the same magnitude. In most practical systems one may represent the particles to a good approximation as solid ellipsoids of revolution. This means that as well two principal optical polarizabilities as two rotational diffusion coefficients are equal. Now $c_{R}(t)$ is given by [19]

$c_{\mathrm{R}}(t)=\sum_{M=-2}^{+2} B_{M} \exp \left(-\left[6 \theta_{\perp}+M^{2}\left(\theta_{\Downarrow}-\theta_{\perp}\right)\right] t\right)$,

where the $B_{\mathrm{M}}$ are those linear combir:ations of the elements of the body fixed polarizability tensor which transform as spherical harmonics of index 2 . $\theta_{1}$ and $\theta_{\perp}$ denote the rotational diffusion coefficient 
around and perpendicular to the symmetry axis respectively. As can be seen from eq. (4), now three relaxation processes can be found in $c_{R}(t)$. If principle axis of optical anisotropy and moments of inertia coincide, eq. (4) reduces to:

$c_{\mathrm{R}}(t)=B \exp \left(-6 \theta_{\perp} t\right)$.

According to Perrin [9] $\theta_{\|}, \theta_{1}$ and $D$ can be expressed in terms of the semi axes $a, b, b$ of the ellipsoid as follows:

$\theta_{1}=\frac{3 k_{\mathrm{B}} T}{16 \pi n a^{3}} \frac{\left(2-\rho^{2}\right) G(\rho)-1}{1-\rho^{4}}$

$\theta_{1}=\frac{3 k_{\mathrm{B}} T}{16 \pi m a^{3}} \frac{1-\rho^{2} G(\rho)}{\left(1-\rho^{2}\right) \rho^{2}}, \quad D=\frac{k_{\mathrm{B}} T}{6 \pi m a} G(\rho)$

$G(\rho)=\left(1-\rho^{2}\right)^{-1 / 2} \ln \left\{\left[1+\left(1-\rho^{2}\right)^{1 / 2}\right] / \rho\right\}$,

$\rho=b / a<1$ prolate ellipsoid,

$G(\rho)=\left(\rho^{2}-1\right)^{-1 / 2} \arctan \left(\rho^{2}-1\right)^{1 / 2}$

$\rho>1$ oblate ellipsoid.

In the limit $\rho \rightarrow 1$, or for a spherical particle we have:

$\theta_{0}=k_{\mathrm{B}} T /\left(8 \pi \eta a^{3}\right), \quad D_{0}=k_{\mathrm{B}} T /(6 \pi \eta a)$,

where $a$ is the hydrodynamic radius of the sphere.

In order to determine from the experiments deviations from a purely spherical shape, we write for $c_{\mathbf{R}}(t)$ :

$c_{\mathrm{R}}(t)=\sum_{M=-2}^{+2} B_{M} \exp \left(-6 \theta_{M} t\right)$,

where one would like to derive $\theta_{M}$ from the experiments by fitting the experimental result to eq. ( 8 ). Note that the value of $D$ can be found from the experimental observation of $c_{\mathrm{vv}}(t)$ (eq. (2)).

From $\theta_{M}$ and $D$ we can derive quantities $V_{M}$ and $V_{\nu}$, defined by

$V_{M}=\left[k_{\mathrm{B}} T / 6 \eta \theta_{M}\right], \quad V_{D}=\left[k_{\mathrm{B}} T / 6 \pi \eta D\right]^{3} 4 \pi / 3$,

where

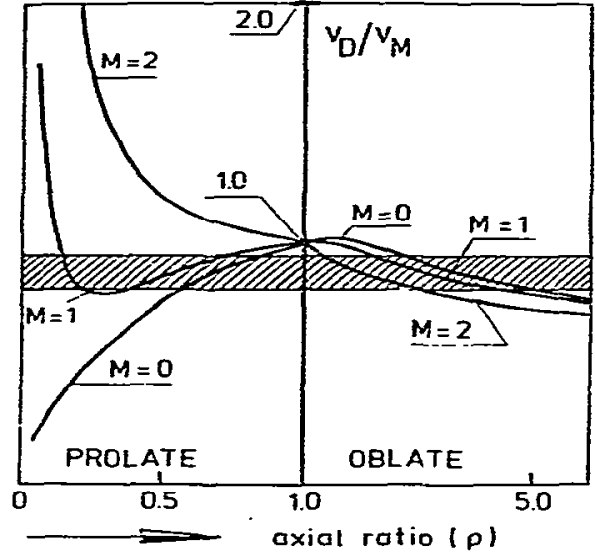

Fig. 3. $V_{D} / V_{M}$ as a function of the axial ratio (cf. eq. (10)). Note that for a spherical shape $V_{\mathrm{D}} / V_{M}=1$ for all values of $M$ (see text).

The so defined quantities $V_{M}$ and $V_{D}$ can be considered as equivalent hydrodynamic volumes for the ellipsoids of revolution considered. It is clear that the ratio $V_{D} / V_{M}=1$ for pure spheres, and deviates from 1 for ellipsoids. In fig. 3 we have plotted the ratio $V_{D} / V_{M}$ as a function of $\rho$ and for $M=0,1$, and 2 . Note that the case $M=0$ corresponds to the true symmetric top molecule [19].

Apart from light scattering from optically aniso. tropic particles, also multiple scattered light may contribute to $\mathrm{VH}$ scattering. Whether the effect of multiple scattering is large depends on the particle concentration. The correlation function of multiple scattered light adds to the correlation function of the anisotropy scattering. Recently there have appeared some papers on this subject [20-24].

In not too concentrated solutions, the main contribution comes from double scattered light. According to Sorensen [20] the correlation function $c_{\mathrm{VH}}(t)$ of double scattering has a slope at $t=0$, which is independent of the scattering angle, and which has the same value as the slope at $t=0$ of the single scattering $\mathrm{VV}$ correlation function at a scattering angle of $180^{\circ}$. The occurrence of double scattered light is a disturbing factor if the characteristic time of the rotational diffusion has the same order of magnitude as the double scattering $[4,5]$. In the case of spheres the different characteristic times can be easily compared with the 


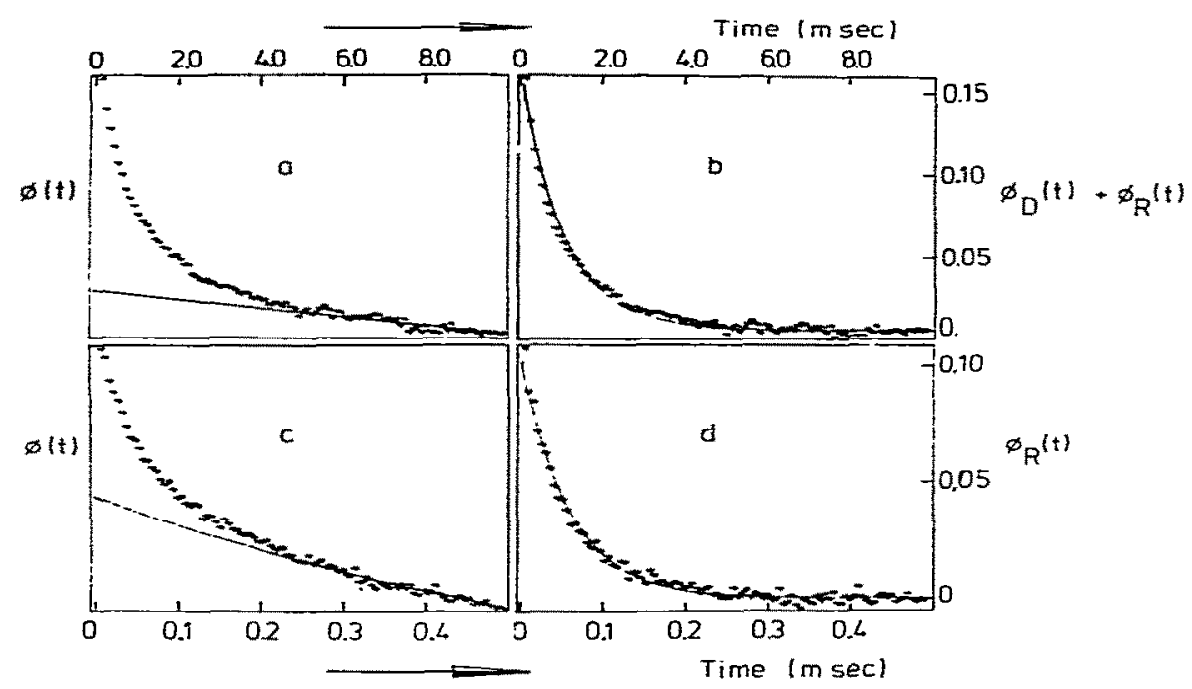

Fig. 4. Subtraction of double scattering contribution and contribution of dust from $\phi(t)$ to obtain $\phi_{\mathrm{R}}(t)$, see text. (a) a linear contribution due to dust is subtracted from $\phi(t)$ to obtain $\phi_{\mathrm{R}}(t)+\phi_{\mathrm{D}}(t)$. (b) From $\phi_{\mathrm{R}}(t)+\phi_{\mathrm{D}}(t)$ one obtains ti . (c) The function $\phi_{\mathrm{S}}(0)+\phi_{\mathrm{D}}(0) \exp \left[-t / \gamma_{\mathrm{D}}\right]$ represented by the drawn line is subtracted from $\phi(t)$ to obtain $\phi_{\mathrm{R}}(t)$. (d) From $\phi_{\mathrm{R}}(t)$ one obtains $\tau \dot{R}$ (see text).

help of eqs. (5) and (7). For rotational diffusion this time is $\tau_{R}=\left(6 \theta_{\perp}\right)^{-1}$ and for double scattering $\tau_{D}$ is the diffusion relaxation time at a scattering angle of $180^{\circ}$ and appears to be: $\tau_{D}=\left[D\left(4 \pi / \lambda_{m}\right)^{2}\right]^{-1}$.

The ratio $\tau_{D} / \tau_{R}$ is:

$\tau_{\mathrm{D}} / \tau_{\mathrm{R}}=9 \lambda_{\mathrm{m}}^{2} /\left(32 \pi^{2} a^{2}\right)$

which means that for $a \approx\left(\lambda_{\mathrm{m}} / 5.5\right) \tau_{\mathrm{D}}=\tau_{\mathrm{R}}$.

The ratio of double $I_{\mathrm{D}}$ to anisotropy $I_{\mathrm{R}}$ scattered intensity can be calculated to be $[20,6]$

$I_{\mathrm{D}} / I_{\mathrm{R}}=\left(2 \pi / \lambda_{\mathrm{m}}\right)^{4}\left(\alpha_{\mathrm{m}}^{4} / \gamma_{\mathrm{m}}^{2}\right) \rho_{\mathrm{N}} F$,

where $\rho_{N}$ is the number density of the scattering particles, $c_{m}$ is the mean polarizability and $\gamma_{m}$ the polarizability anisotropy [6] of the particles in the medium. $F$ is a function of the geometrical set-up of the experiment and varies slightly with scattering angle.

\section{Results}

The correlator determines the function $\dot{\varphi(t)}$, cf. eq. (1). The inspection of the experimental results (figs. 1 and 2) made us decide to try to fit the depolarized correlation functions to the sum of three exponential functions:

$$
\begin{aligned}
\phi(t) & =\phi_{\mathrm{R}}(t)+\phi_{\mathrm{D}}(t)+\phi_{\mathrm{S}}(t) \\
= & \phi_{\mathrm{R}}(0) \exp \left[-t / \tau_{\mathrm{R}}^{\prime}\right]+\phi_{\mathrm{D}}(0) \exp \left[-t / \tau_{\mathrm{D}}^{\prime}\right] \\
+ & \phi_{\mathrm{S}}(0) \exp \left[-t / \tau_{\mathrm{S}}^{\prime}\right],
\end{aligned}
$$

where $\phi_{\mathrm{R}}(0), \phi_{\mathrm{D}}(0), \phi_{\mathrm{S}}(0)$ and $\tau_{\mathrm{R}}^{\prime}, \tau_{\mathrm{D}}^{\prime}, \tau_{\mathrm{S}}^{\prime}$ are adjustable parameters with $\tau_{S}^{\prime} \gg \tau_{D}^{\prime} \gg \tau_{R}^{\prime}[25]$.

The procedure to evaluate these parameters is as follows: For each experimental situation (this means at fixed $\theta, \eta$ and concentration) we measured two correlation functions at different time scales. In the longest time scale correlation function the interval time was chosen in such a way that beyond the first few points the function can be represented by $\phi_{D}(t)+\phi_{\mathrm{S}}(t)$. From this function (see fig- 4a) we subtracted a linear contribution. This contribution represents $\phi_{S}(0) \exp \left[-t / \tau_{S}^{\prime}\right] \approx \phi_{S}(0)\left[1-t / \tau_{S}^{\prime}\right]$ for $t \leqslant \tau_{S}^{\prime}$. The resulting function (see fig. $4 \mathrm{~b}$ ) is beyond the first few points a good approximation of $\phi_{\mathcal{D}}(t)$. To this part of $\phi_{D}(t)$ we applied the cumulant method [26] to obtain $\tau_{D}^{\prime}$ (drawn line fig. 4b).

The value of $\tau_{D}^{\prime}$ is used to analyze the short time 
Table 1

Values of parameters used to fit the experimental data and orientational correlation times of ribosomal particles. (a) The same number corresponds to the same experiment. (b) Diffusion coefficient obtained from eq. (9) and $V_{D}$ obtained from eq. (10). (c) Values for $t_{D}$ in function $\phi_{S}(0)+\phi_{D}(0)$ exp $\left[-t / \tau_{D}^{\prime}\right]$ to be subtracted from $\phi_{R}(t)$ (see text). (d) t $\tau_{R}$ is correlation time of $\phi_{R}(t)$; the value between brackets is correlation time of best fit exponential function, the other value is determined by curnulant method. (e) cf. section 4. (f) Correlation time calculated from eq. (17) and neglecting contribution due to number fiuctuations (see text). (g) As f from eq. (17) but by putting $I_{S}=0$. (h) (hydrodynamic volume) ${ }^{-1}$ calculated from eq. (19) and $\tau_{R}$ in $f$. (i) As $h$ but using $\tau_{R}$ in $g$. $(j, k)$ Ratio of hydrodynamic volumes from effective translational and rozational diffusion coefrictents $(\mathbf{i} \rightarrow \mathbf{h}$ and $\mathbf{k} \rightarrow \mathbf{i})$.

\begin{tabular}{|c|c|c|c|c|c|c|c|c|c|c|c|c|}
\hline $\begin{array}{l}\text { (a) } \\
\text { No. }\end{array}$ & $\begin{array}{l}7 \\
\left(10^{-3}\right. \\
\left.\mathrm{Nm}^{-1} \mathrm{~s}^{-1}\right)\end{array}$ & $\begin{array}{l}\text { (b) } \\
D\left(V_{\mathrm{D}}\right)\end{array}$ & $\begin{array}{l}\text { (c) } \\
\text { to } \\
\text { (us) }\end{array}$ & $\begin{array}{l}\text { (d) } \\
\tau_{R}^{\prime} \\
\text { (us) }\end{array}$ & $\begin{array}{l}\text { (e) } \\
\frac{\phi D^{(0)}}{\phi S(0)}\end{array}$ & $\begin{array}{l}(\mathrm{e}) \\
\frac{\phi_{\mathrm{D}}(0)}{\phi_{R}(0)}\end{array}$ & $\begin{array}{l}(f) \\
\text { TR } \\
(\mu \mathrm{s})\end{array}$ & $\begin{array}{l}\text { (g) } \\
\tau_{R} \\
\text { (us) }\end{array}$ & $\begin{array}{l}\text { (n) } \\
(1 / \mathrm{W} \\
10^{-5} \\
\left(\mathrm{~nm}^{-3}\right)\end{array}$ & $\begin{array}{l}\text { (i) } \\
(1 / V) \\
10^{-5} \\
\left(\mathrm{~nm}^{-3}\right)\end{array}$ & $\begin{array}{l}\text { (j) } \\
V_{\mathbf{D}} \\
\mathbf{a} / \mathrm{V},\end{array}$ & $\begin{array}{l}(\mathbf{k}) \\
V_{\mathbf{D}} \\
(1 / V)\end{array}$ \\
\hline $\begin{array}{l}1 \\
2 \\
1 \\
2\end{array}$ & $\left.\begin{array}{l}19.6 \\
19.6 \\
19.6 \\
19.6\end{array}\right)$ & $\begin{array}{l}8.61 \times \\
10^{-13} \mathrm{~m}^{2} \mathrm{~s}^{-1} \\
\left(8760 \mathrm{~nm}^{3}\right)\end{array}$ & $\begin{array}{l}600 \\
600 \\
800 \\
800\end{array}$ & $\begin{array}{l}45(48) \\
43(46) \\
43(53) \\
42(51)\end{array}$ & $\begin{array}{l}1.11 \\
0.41 \\
1.53 \\
0.49\end{array}$ & $\begin{array}{l}1.50 \\
1.54 \\
1.61 \\
1.55\end{array}$ & $\begin{array}{l}49 \\
45 \\
47 \\
44\end{array}$ & $\begin{array}{l}53 \\
51 \\
50 \\
49\end{array}$ & $\begin{array}{r}9.8 \\
10.6 \\
10.2 \\
10.9\end{array}$ & $\begin{array}{l}9.1 \\
9.5 \\
9.6 \\
9.8\end{array}$ & $\begin{array}{l}0.86 \\
0.93 \\
0.90 \\
0.95\end{array}$ & $\begin{array}{l}0.79 \\
0.83 \\
0.84 \\
0.86\end{array}$ \\
\hline $\begin{array}{l}3 \\
4 \\
3 \\
4\end{array}$ & $\left.\begin{array}{l}1.44 \\
1.44 \\
1.44 \\
1.44\end{array}\right)$ & $\begin{array}{l}1.26 \times \\
10^{-11} \mathrm{~m}^{2} \mathrm{~s}^{-1} \\
\left(7400 \mathrm{~nm}^{3}\right)\end{array}$ & $\begin{array}{l}50 \\
50 \\
70 \\
70\end{array}$ & $\begin{array}{l}3.4(3.6) \\
3.7(4.8) \\
3.8(4.8) \\
4.3(5.3)\end{array}$ & $\begin{array}{l}0.78 \\
0.49 \\
1.00 \\
0.60\end{array}$ & $\begin{array}{l}3.24 \\
3.10 \\
3.24 \\
3.11\end{array}$ & $\begin{array}{l}3.5 \\
3.8 \\
4.0 \\
4.5\end{array}$ & $\begin{array}{l}3.8 \\
4.2 \\
4.3 \\
4.9\end{array}$ & $\begin{array}{r}10.0 \\
9.2 \\
8.9 \\
7.9\end{array}$ & $\begin{array}{l}9.3 \\
8.5 \\
8.3 \\
7.3\end{array}$ & $\begin{array}{l}0.74 \\
0.68 \\
0.66 \\
0.59\end{array}$ & $\begin{array}{l}0.69 \\
0.63 \\
0.61 \\
0.54\end{array}$ \\
\hline $\begin{array}{l}5 \\
6 \\
7 \\
5 \\
6 \\
7\end{array}$ & $\left.\begin{array}{l}7.31 \\
7.31 \\
7.31 \\
7.31 \\
7.31 \\
7.31\end{array}\right)$ & $\begin{array}{l}2.25 \times \\
10^{-12} \mathrm{~m}^{2} \mathrm{~s}^{-1} \\
\left(9470 \mathrm{~nm}^{3}\right)\end{array}$ & $\begin{array}{l}150 \\
150 \\
150 \\
250 \\
250 \\
250\end{array}$ & $\begin{array}{l}18(11) \\
18(12) \\
18(12) \\
15(17) \\
15(18) \\
13(18)\end{array}$ & $\begin{array}{l}0.035 \\
0.031 \\
0.0271 \\
0.036 \\
0.030 \\
0.027\end{array}$ & $\begin{array}{l}1.33 \\
1.42 \\
1.37 \\
1.30 \\
1.36 \\
1.19\end{array}$ & $\begin{array}{l}18 \\
19 \\
18 \\
15 \\
15 \\
13\end{array}$ & $\begin{array}{l}21 \\
22 \\
22 \\
18 \\
18 \\
15\end{array}$ & $\begin{array}{r}9.9 \\
9.7 \\
9.8 \\
11.9 \\
11.9 \\
13.7\end{array}$ & \begin{tabular}{|r|}
8.4 \\
8.2 \\
8.3 \\
10.1 \\
10.1 \\
11.6
\end{tabular} & $\begin{array}{l}0.94 \\
0.91 \\
0.93 \\
1.13 \\
1.13 \\
1.3\end{array}$ & $\begin{array}{l}0.79 \\
0.77 \\
0.78 \\
0.95 \\
0.96 \\
1.10\end{array}$ \\
\hline
\end{tabular}

scale correlation function, which can be represented by $\phi_{\mathrm{R}}(t)+\phi_{\mathrm{D}}(t)+\phi_{\mathrm{S}}(0)$ as $t<\tau_{\mathrm{D}}^{\prime} \ll \tau_{\mathrm{S}}^{\prime}$. The wing of this correlation function can therefore be fitted to $\dot{\phi}_{\mathrm{D}}(t)+\dot{\phi}_{\mathrm{S}}(0)$ (drawn line fig. 4c). The function $\mathrm{ob}$ tained after subtraction of the drawn line represents $\Phi_{\mathrm{R}}(t)$ (fig. 4d). We have applied the cumulant method to obtain $\tau_{D}^{\prime}$. The correlation time which has been determined from a least squares fit of $\phi_{R}(t)$ to an exponeiatial is somewhat larger than $\tau_{R}^{\prime}$. The value obtained for $\tau_{R}^{\prime}$ is insensitive to the value for $\tau_{D}^{\prime}$. The value found for $\phi_{S}(0)$ is different in every experiment. The value $\phi_{D}(0) / \phi_{R}(0)$, however, varies only slightly for the different scattering angles used, but is dependent on the concentration. The results are summarized in table 1.

In fig. 5 we have also plotted $\tau_{D}^{\prime}$ and $D^{-1}$ versus the viscosity. The linear behaviour found is in accordance with theory. At even higher viscosities $\left(\eta=48.6 \times 10^{-3}\right.$ $\mathrm{g} \mathrm{m}^{-1} \mathrm{~s}^{-1}$ ) we observed a deviation from linearity which we have found to be caused by dissociation of the ribosomal particle.

\section{Discussion}

The depolarized scatiered intensity is expected to contain contributions from the anisotropy and multiple scattered light from the ribosomes, and also contributes from dust, solvent and cell windows. It has been experimentally verified that the last two contributions can be neglected for the ribosomal concentrations used.

From the turbidity of the most concentrated solutions the optical mean free path [22] appeared to be $10 \mathrm{~cm}$. This means that, from the multiple scattering, only the double scattering contribution is of significance.

Therefore the correlation function of the depolarized scattered field $c_{\mathrm{VH}}(t)$ can be considered as a sum of the correlation functions of the anisotropy scattered field $c_{R}(t)$, the double scattered field $c_{D}(t)$ and of the field scattered from dust $c_{\mathrm{S}}(t)$.

The experimentally observed correlation function $\phi(t)$ therefore assumes the form $[14,25]$ 


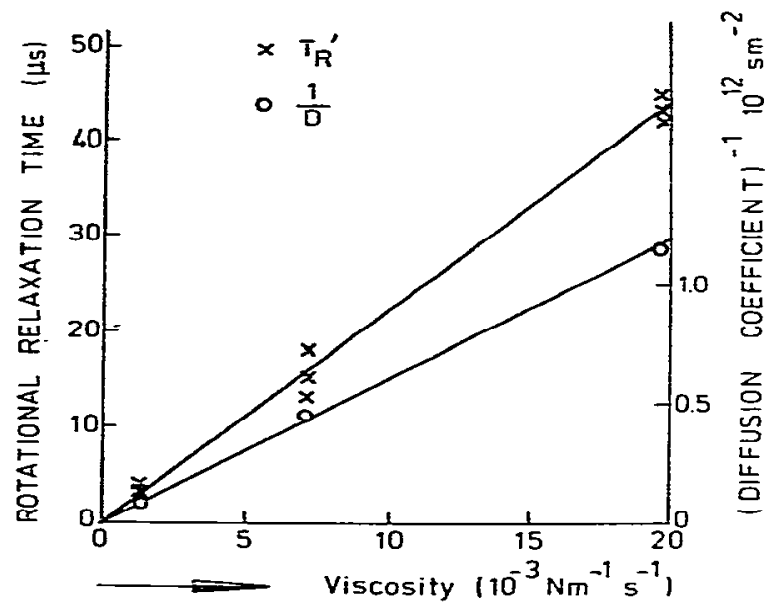

Fig. 5. The cumulant relaxation time $\tau_{R}^{\prime}$ and $D^{-1}$ versus viscosity. As expected both quantities depend linearly on the viscosity.

$\phi(t)=\sum_{i, j}^{i} I_{i} I_{j} c_{i}(t) c_{j}(t)$,

where $i$ and $j$ can be $\mathbf{R}, \mathbf{D}$ and $S$. In order to determine which of the nine terms in the sum of eq- (15) belong to $\phi_{\mathrm{R}}(t), \phi_{\mathrm{D}}(t)$ and $\dot{\phi}_{\mathrm{S}}(t)$, we estimate the correlation times of the functions $c_{\mathrm{R}}(t), c_{\mathrm{D}}(t)$ and $c_{\mathrm{S}}(t)$ by taking a spherical model for the ribosomal particle. From the translational diffusion experiments [2] one can calculate the Stokes radius of the particle $(12.6 \mathrm{~nm})$. According to eq. (12) one finds that $\tau_{\mathrm{D}} / \tau_{\mathrm{R}} \approx 30$ for $\lambda=514.5 \mathrm{~nm}$. We assume that the contribution to $c_{S}(t)$ arises due to the anisotropy of the dust particles. Assuming that the dust particles have a radius ten times as large as the ribosomes, one finds that $\tau_{S} / \tau_{R} \approx 1000$. Therefore we arrive at the conclusion that $\tau_{S} \ll \tau_{D} \ll \tau_{S}$. Now the sum of the nine terms in eq. (15) can be grouped according to their relaxation times. This means that:

$$
\begin{aligned}
& \phi_{\mathrm{S}}(t)=I_{\mathrm{S}}^{2} c_{\mathrm{S}}^{2}(t)+\left\langle\delta N_{\mathrm{S}}(0) \delta N_{\mathrm{S}}(t)\right\rangle \\
& \phi_{\mathrm{D}}(t)=2 I_{\mathrm{S}} I_{\mathrm{D}} c_{\mathrm{S}}(t) c_{\mathrm{D}}(t)+I_{\mathrm{D}}^{2} c_{\mathrm{D}}^{2}(t) \\
& \phi_{\mathrm{R}}(t)=2 I_{\mathrm{S}} I_{\mathrm{R}} c_{\mathrm{S}}(t) c_{\mathrm{R}}(t) \\
& \quad+2 I_{\mathrm{D}} I_{\mathrm{R}} c_{\mathrm{D}}(t) c_{\mathrm{R}}(t)+I_{\mathrm{R}}^{2} c_{\mathrm{R}}^{2}(t)
\end{aligned}
$$

where $\left\langle\delta N_{\mathrm{S}}(0) \delta N_{\mathrm{S}}(t)\right)$ represents the contribution due to number fluctuations of dust particles [27].
We have found that $\phi_{S}(0)$ varies for every experiment in agreement with the assumption that dust occasionally contributes to the depolarized scattering intensity. We have also found that $\phi_{D}(0) / \phi_{R}(0)$ varies about $20 \%$ independent of $\phi_{S}(0)$. As has been stated in eq. (13) $I_{\mathrm{D}} / I_{\mathrm{R}}$ is constant for fixed $\rho_{\mathrm{N}}$ and $\theta$.

As can be seen from eqs. (14) and (16) the experimental value of $\tau_{D}$ depends on the occasional flares of dust occurring during the experiment. Therefore we have used two extreme experimental values for $\tau_{D}^{\prime}$ to analyse the short time correlation function. By taking these values of $\tau_{\mathrm{D}}^{\prime}$ we found, however, that the $t \rightarrow 0$ slope of $\phi_{\mathrm{R}}(t)$ is rather insensitive to this value (cf. fable 1 , column (d)). From this initial slope $\tau_{R}^{\prime}$ can be determined. From eqs. (14) and (16c) we now find from the limit $t \rightarrow 0$ :

$$
\begin{gathered}
\tau_{\mathrm{R}}^{-1}=\frac{2 I_{\mathrm{S}}+2 I_{\mathrm{D}} \div I_{\mathrm{R}}}{2 I_{\mathrm{S}}+2 I_{\mathrm{D}}+2 I_{\mathrm{R}}}\left(\tau_{\mathrm{R}}^{\prime}\right)^{-1} \\
-\frac{I_{\mathrm{D}}}{I_{\mathrm{S}}+I_{\mathrm{D}}+I_{\mathrm{R}}} \tau_{\mathrm{D}}^{-1} .
\end{gathered}
$$

The procedure of determining the values of the quantities in eq. (17) from eq. (16) and the experimental values for $\phi_{S}(0), \phi_{D}(0)$ and $\phi_{R}(0)$ anid also $\tau_{D}^{\prime}$ and $\tau_{R}^{\prime}$ goes along two extreme cases: $i)$ the number fluctuations in $\phi_{\mathrm{S}}(0)$ are dominant: i.e. $I_{\mathrm{S}}=0$; ii) the number fluctustions can be neglected: i.e. $\phi_{S}(0)=I_{S}^{2}$.

Ad i) From $\phi_{D}(0) / \phi(0)$ (table 1) $I_{D} / I_{R}$ can be determined by using eqs. $(16 \mathrm{~b}, \mathrm{c})$. By noting that in this case $\tau_{D}=\tau_{D}^{\prime} / 2$ one finds from eq. (17) $\tau_{\mathrm{R}}$ (table 1 , column $(\mathrm{g})$ ).

Ad ii) From $\phi_{D}(0) / \phi_{S}(0)$ and $\phi_{D}(0) / \phi_{R}(0)$ one finds with eqs. $(16 \mathrm{a}, \mathrm{b}, \mathrm{c}) I_{\mathrm{S}} / I_{\mathrm{D}}$ and $I_{\mathrm{S}} / I_{\mathrm{R}}$ - By applying the cumulant method to eq. $(16 b)$ one finds $\tau_{D}$ from $\tau_{D}^{\prime}$. As before, now $\tau_{R}$ can be found with eq. (17). See table 1, column (f).

The actual value of $\tau_{R}$ is in between the limits of columns (f) and (g) of table 1 . In spite of the complex method of fitting the correlation functions, the correlation times can be determined with good reliability. This is a consequence of the relative large differences between $\tau_{D}$ and $\tau_{R}$.

Now we shall turn to the meaning of $\tau_{R}$ in terms of the orientational moments of inertia. From the first cumulant of eq. (8) one finds: 
$\frac{1}{\tau_{\mathrm{R}}}=\sum_{M=-2}^{+2} 6 B_{M} \theta_{M} /\left(\sum_{M=-2}^{+2} B_{M}\right)$

Combining eqs. (9) and (18) gives:

$$
\begin{aligned}
\tau_{\mathrm{R}}^{-1} & =\frac{k_{\mathrm{B}} T}{\eta}\left(\sum_{M=-2}^{+2} B_{M} / V_{M}\right) /\left(\sum_{M=-2}^{+2} B_{M}\right) \\
& =\frac{k_{\mathrm{B}} T}{\eta}\langle 1 / V\rangle .
\end{aligned}
$$

This means that $\tau_{\mathbf{R}}^{-1}$ gives a weighted average reciprocal volume. In table 1 the value $\langle 1 / V\rangle$, for the two extreme cases of $\tau_{R}$ considered, are given. The mean value $\langle 1 / V\rangle$ of $1 / V$ over the experiments performed at $\eta=19.6 \mathrm{~g} \mathrm{~m}^{-1} \mathrm{~s}^{-1}$ turns out to be $(10.4 \pm 0.5)$ $\times 10^{-5} \mathrm{~nm}^{-3}$ in the "absence" of number fluctuations, and $(9.5 \pm 0.3) \times 10^{-5} \mathrm{~nm}^{-3}$ for the case that we assumed $I_{\mathrm{S}}=0$. In order to decide whether these experiments contain information about the deviation from the spherical shape, we have also to consider $V_{D}$, as found from translational diffusion experiments (cf. table 1). The value of $V_{D^{\bullet}}\langle 1 / V\rangle$ for the measurements at $\eta=19.6 \mathrm{~g} \mathrm{~m}^{-1} \mathrm{~s}^{-1}$ is found to be in the range: $0.87 \pm 0.07$ if we consider both methods of evaluating $\tau_{R}$. This range is indicated in fig. 3 by the shaded area. As can be seen, we have clear indication that the particle's shape deviates from a sphere. It has been suggested [28], that one may conclude from electron microscopy studies [29] that the 70S ribosume can be represented by an oblate ellipsoid. In this case one concludes from our experiments (based on the most reliable experiments at $\eta=19.6 \mathrm{cps}$ ) that that axial ratio will not exceed 5 (cf. fig. 3 ), in agreement with the result that $\rho<1.5$ obtained from fluorescent depolarization studies [28]. In order to perform accurate experiments at low viscosities a correlator operating in the real time mode (rather than in the batch mode) at the desired sampling times is required.

In the model discussed above, we considered the ribosome as a solid ellipsoid of revolution. In order to justify the conclusion drawn regarding the shape of the particle we considered the effect of free draining. The most extreme case one may coinsider is a ribosome consisting of a number of independent spherical subunits. In our case this number is 58 being the number of proteins and RNA particles constituting the ribosome.
If we consider these subunits to be spheres of 2.2 nm radius, then their total volume equals the dry volume of the ribosome. On this basis we calculated the ratio of the volumes found from translation and rotation diffusion, assuming a spherical shape for the ribosome, from eqs. (5.6) and (5.11) of the paper of Deutch and Felderhof [11]. This ratio appeared to be 0.90 . This is, however, a very extreme case since we considered only the smallest possible number of subunits, which were moreover supposed to be spherical and non hydrated. For less extreme cases the ratio approaches 1 . Therefore we believe that our conclusion that the particles deviate from spherical shape remains valid. This conclusion could however be impaired by the effect of free draining. In conclusion we shall summarize our results as follows. The method of photon correlation spectroscopy has proved its usefulness, parallel to other techniques as electron microscopy, X-ray scattering and fluorescence studies, in the hydrodynamic characterization of ribosomal particles. Especially in the case of depolarized scattering the occurrence of double scattering can give problems if rotational relaxation times (nearly) coincide with double scattering relaxation.

As in our case, the scattering phenomenon may be complex. This means that a careful analysis of the experiments is desired. If this is done, reliable results can be obtained.

\section{Acknowlergements}

The authors thank Drs. H. Goumans for his help purifying the ribosomal particles and Professor Dr. H.O. Voorma, Professor Dr. A. Vrij and Dr. W.J.C. Amesz for stimulating discussions. We thank H.J. Mos for his technical assistance.

\section{References}

[1] B.J. Berne and R. Pecora, Ann. Rev. Phys. Chem. 25 (1974) 233.

[2] D.E. Koppel, Biochem. 13 (1974) 2712.

[3] S.B. Dubin, N.A. Clark and G.B. Benedek, J. Chem. Phys. 54 (1971) 5158 .

[4] T.A. Xing, A. Knox and J.D.C. McAdam, Biopolym. 12 (1973) 1917.

[5] J.M. Schurr and K.S. Schmitz, Biopolym 12 (1973) 1021. 
[6] S.B. Dubin, Methods of Enzy mology XXVIIc (1973) 119.

[7] B.J. Berne and R. Pecora, Dynamic light scattering (John Wiley, New York, 1976) chapter VII-3.

[8] P. Debye, Polar molecales (Dover, New York, 1929).

[9] F. Perrin, J. de Phys. et Rad. V (1934) 497; VII (1936) 1.

[10] B.U. Felderhof and J.M. Deutch, J. Chem. Phys. 62 (1975) 2391.

[11] 3.M. Deutch and B.U. Felderhof, J. Chem. Phys. 62 (1975) 2398.

[12] N. Naaktgeboren, A. Vermaas and H.O. Voorma, Eur. J. Blochem. 75 (1977) 455.

[13] W.E. Hill, G.P. Rosetti and K.E. van Holde, J. Mol. Biol. 44 (1969) 263.

[14] B.J. Berne and R. Pecora, Dynamic light scattering (John Wiley, New York, 1976) Chapter IV B-3.

[15] R. Pecora, J. Chem. Phys. 40 (1964) 1604.

[16] Y.H. Lin and C.H. Wang, J. Chem. Phys. 66 (1977) 5578.

[17] D.R. Bauer, J.I. Brauman and R. Pecora, Macromolecuies 8 (1975) 443 .

[18] D. Favro, Phys. Rev. 119 (1960) 53.
[19] B.J. Berne and R. Pecora, Dynamic light scattering (John Wiley, New York, 1976) Chapter VII-5.

[20] C.M. Sorensen, R.C. Mackler and W.J. O'Sullivan, Phys. Rev. A14 (1976) 1520 .

[21] A. Bфe and T. Sikkeland, Phys. Rev. A16 (1977) 2105.

[22] D.B. Siano, B.J. Berne and G.W. Fiynn, J. Coll. Int. Sci. $63(1978) 282$.

[23] F.C. van Rijswijk and U.L. Smith, Physica 83A (1976) 121.

[24] D.K. Kim, J.G. Callagher and C.D. Armeniades, Phys. Rev, Leit. 39 (1977) 804.

[25] F.C. Chen, A. Yeh and B. Chu, J. Chem. Phys. 66 (1977) 1290.

[26] D. Koppel, J. Chem. Phys. 57 (1972) 4814.

[27] D.W. Schaefer and B.J. Berne, Phys. Rev. Lett. 28 (1972) 475.

[28] B. Amand, F. Pochon and D. Lavalette, Biochimie 59 (1977) 779.

[29] J.A. Lake, J. Mol. Biol. 105 (1976) 131. 